\title{
Efficacy of sertraline against Trypanosoma cruzi: an in vitro and in silico study
}

\author{
Daiane Dias Ferreira', Juliana Tonini Mesquita' ${ }^{1}$ Thais Alves da Costa Silva ${ }^{1}$, Maiara Maria Romanelli', \\ Denise da Gama Jaen Batista ${ }^{2}$, Cristiane França da Silva ${ }^{2}$, Aline Nefertiti Silva da Gama², Bruno Junior Neves ${ }^{3}$, \\ Cleber Camilo Melo-Filho ${ }^{3}$, Maria de Nazare Correia Soeiro ${ }^{2}$, Carolina Horta Andrade ${ }^{3}$ \\ and Andre Gustavo Tempone ${ }^{1 *}$ (i)
}

\begin{abstract}
Background: Drug repurposing has been an interesting and cost-effective approach, especially for neglected diseases, such as Chagas disease.

Methods: In this work, we studied the activity of the antidepressant drug sertraline against Trypanosoma cruzi trypomastigotes and intracellular amastigotes of the $Y$ and Tulahuen strains, and investigated its action mode using cell biology and in silico approaches.

Results: Sertraline demonstrated in vitro efficacy against intracellular amastigotes of both $T$. cruzi strains inside different host cells, including cardiomyocytes, with $\mathrm{IC}_{50}$ values between 1 to $10 \mu \mathrm{M}$, and activity against bloodstream trypomastigotes, with $\mathrm{IC}_{50}$ of $14 \mu \mathrm{M}$. Considering the mammalian cytotoxicity, the drug resulted in a selectivity index of 17.8. Sertraline induced a change in the mitochondrial integrity of $T$. cruzi, resulting in a decrease in ATP levels, but not affecting reactive oxygen levels or plasma membrane permeability. In silico approaches using chemogenomic target fishing, homology modeling and molecular docking suggested the enzyme isocitrate dehydrogenase 2 of $T$. cruzi $(T \mathrm{CIDH} 2)$ as a potential target for sertraline.
\end{abstract}

Conclusions: The present study demonstrated that sertraline had a lethal effect on different forms and strains of $T$. cruzi, by affecting the bioenergetic metabolism of the parasite. These findings provide a starting point for future experimental assays and may contribute to the development of new compounds.

Keywords: Trypanosoma cruzi, Drug, Treatment, Sertraline, Drug repurposing, Drug repositioning

\section{Background}

Chagas disease is a neglected tropical protozoan disease that affects 8 million people, mainly in South and Central America [1]; however, cases have also been reported in North America, Europe and some other Western countries [2]. The current treatment of Chagas disease is an alarming issue. A recent clinical study re-evaluated the efficacy of benznidazole (BZ), the only available treatment in Brazil; despite a reduction in parasitemia, the study revealed that BZ induced no significant improvements in cardiac clinical outcomes during chronic-phase Chagas disease [3]. According to the

\footnotetext{
*Correspondence: andre.tempone@ial.sp.gov.br; atempone@usp.br ${ }^{1}$ Instituto Adolfo Lutz, Centre for Parasitology and Mycology, Avenida Dr. Arnaldo 351, $8^{\circ}$ andar, sala 9, CEP, São Paulo, SP 01246-000, Brazil Full list of author information is available at the end of the article
}

non-profit organization Drugs for Neglected Diseases initiative (DNDi), in the next 5 years, 200,000 people living with Chagas disease will die from heart disease and related complications. DNDi also highlighted the urgent need to find better treatments [4]. Additionally, the drug safety of the available treatment is inadequate; $\mathrm{BZ}$ is implicated in serious systemic side effects, including anorexia, vomiting, peripheral polyneuropathy, depression of bone marrow, and allergic dermopathy. As a consequence of these adverse reactions, treatment has to be discontinued [5].

The discovery of new therapeutic agents is expensive and may take many years. Several strategies have been implemented in order to reduce the costs and time of the drug discovery process. Drug repositioning has emerged as a promising strategy for drug discovery for

(C) The Author(s). 2018 Open Access This article is distributed under the terms of the Creative Commons Attribution 4.0 International License (http://creativecommons.org/licenses/by/4.0/), which permits unrestricted use, distribution, and 
Neglected Tropical Diseases (NTDs). Also referred to as drug repurposing, this strategy consists of finding novel indications for approved drugs [6]. Sertraline (SERT), a serotonin reuptake inhibitor, is widely used in the treatment of patients with depression and severe anxiety disorders [7], and has exhibited antifungal [8], antimicrobial [9], and anticancer activities [10]. The drug has shown promising activity against the protozoan Leishmania donovani; it reduced the parasite burden of animals by $72 \%$ at doses of $10 \mathrm{mg} / \mathrm{kg}$ for 30 days [11].

In the present work, we evaluated the activity of SERT against T. cruzi trypomastigotes and intracellular amastigotes of the $\mathrm{Y}$ and Tulahuen strains and investigated its mode of action using cell biology and in silico chemogenomic approaches.

\section{Methods}

Additional file 1 shows a Flowchart of the global methodology.

\section{Animals}

$\mathrm{BALB} / \mathrm{c}$ mice were supplied by the animal breeding facility at the Adolfo Lutz Institute of São Paulo whereas Swiss mice were obtained from the Fundação Oswaldo Cruz (FIOCRUZ) Rio de Janeiro. The mice were maintained in sterilized cages under a controlled environment and received water and food ad libitum. Animal procedures were performed with the approval of the Research Ethics Commission, in agreement with the Guidelines for the Care and Use of Laboratory Animals from the National Academy of Sciences. All procedures carried out at Institute Adolfo Lutz were approved by the Committee for Ethics in the Use of Animals (CEUA 04/2016). All procedures performed at FIOCRUZ were in accordance with the guidelines established by the FIOCRUZ Committee for Ethics in the Use of Animals (CEUA LW16/14).

\section{Drugs and chemicals}

Resazurin, Roswell Park Memorial Institute medium (RPMI 1640) without phenol red, and Hanks' Balanced Salt Solution (HBSS), were purchased from Sigma-Aldrich. Sytox Green ${ }^{\circ}$ and H2CDFDA (2 ', 7'-dichlorodihydrofluorescein diacetate) were purchased from Molecular Probes ${ }^{-}$(Invitrogen ${ }^{\mathrm{m}}$ ). Fetal Bovine Serum (FBS) was obtained from Gibco and gentamicin sulfate from Hipolabor Pharmaceuticals. Benznidazole (BZ) and sertraline (SERT) were purchased from Sigma-Aldrich. All other reagents not mentioned were purchased from Sigma-Aldrich.

\section{Parasites and mammalian host cells}

\section{$T$. cruzi ( $Y$ strain - culture trypomastigotes)}

Trypomastigotes were maintained in LLC-MK2 cells with RPMI-1640 medium supplemented with $2 \%$ fetal bovine serum (FBS) at $37{ }^{\circ} \mathrm{C}$ and $5 \% \mathrm{CO}_{2}$ in a humidified incubator.

\section{T. cruzi ( $Y$ strain - bloodstream trypomastigotes - BT)}

Trypomastigotes were obtained from the blood samples of infected albino Swiss mice at the peak of parasitemia. The purified parasites were resuspended in Dulbecco's Modified Eagle Medium (DMEM) supplemented with $10 \%$ FBS as reported previously [12].

\section{Macrophages}

Macrophages used in the intracellular amastigote assays were collected from the peritoneal cavity of BALB/c mice by washing with RPMI-1640 medium supplemented with $10 \%$ FBS and maintained at $37{ }^{\circ} \mathrm{C}$ in an atmosphere of $5 \% \mathrm{CO}_{2}$ in a humidified incubator.

\section{Cardiac cell cultures (CC)}

Cardiac cells were used in the cytotoxicity and intracellular amastigote assays. Primary cultures of embryonic cardiac cells were obtained from Swiss mice as previously reported [12]. Briefly, after purification, the CC were seeded at a density of $\left(0.2 \times 10^{6}\right.$ cell/well $)$ into 24-well microplates containing gelatin-coated cover slips as previously described. The cardiac cultures were then sustained at $37{ }^{\circ} \mathrm{C}$ in DMEM supplemented with $10 \%$ horse serum, $5 \%$ fetal bovine serum, $2.5 \mathrm{mM} \mathrm{CaCl}_{2}$, $1 \mathrm{mM}$ L-glutamine and $2 \%$ chicken embryo extract.

\section{NCTC cells-clone $L 929$}

NCTC cells were maintained in medium M-199 supplemented with $10 \%$ FBS and were maintained at $37{ }^{\circ} \mathrm{C}$ under $5 \% \mathrm{CO}_{2}$ in a humidified incubator.

\section{Determination of the Anti-T. cruzi activity Culture trypomastigotes}

To determine the $50 \%$ inhibitory concentration $\left(\mathrm{IC}_{50}\right)$ against T. cruzi, trypomastigotes were counted in a Neubauer hemocytometer and seeded at $\left(1 \times 10^{6}\right.$ cells/well $)$ in 96-well microplates. The drug was dissolved in dimethyl sulfoxide (DMSO), diluted with RPMI-1640 medium at different concentrations for $24 \mathrm{~h}$ at $37^{\circ} \mathrm{C}$ and placed in a $5 \% \mathrm{CO}_{2}$ humidified incubator. The parasite viability was determined using the colorimetric resazurin assay [13]. Benznidazole was used as the standard drug. The optical density was read at $570 \mathrm{~nm}$ (FilterMax F5 Multi-Mode Microplate Reader, Molecular Devices). DMSO was used at a maximal concentration of $0.5 \%$ in all assays and was incubated with cells as an internal control.

\section{Bloodstream trypomastigotes (BT)}

The trypomastigotes $\left(5 \times 10^{6} / \mathrm{mL}\right)$ were incubated for $24 \mathrm{~h}$ at $37^{\circ} \mathrm{C}$ in RPMI medium in the presence or absence of serial dilutions of the drug $(0$ to $50 \mu \mathrm{M})$. After 
$24 \mathrm{~h}$ of incubation, the parasite death rate was determined by light microscopy through direct quantification of the number of living parasites using a Neubauer chamber, and the $\mathrm{IC}_{50}$ was then calculated.

\section{Determination of cytotoxicity Cytotoxicity against mammalian cells} NCTC cells $929\left(6 \times 10^{4}\right.$ cells/well $)$ in 96-well microplates were incubated with the drug for $48 \mathrm{~h}$ at $37^{\circ} \mathrm{C}$ in a $5 \% \mathrm{CO}_{2}$ incubator. The selectivity index (SI) was determined using the following formula: $\mathrm{CC}_{50}$ against mammalian cells/ $\mathrm{IC}_{50}$ against parasites. The cell viability was determined using the colorimetric resazurin assay [13]. The obtained data represent the mean of two independent experiments performed in duplicate.

\section{Cytotoxicity against CC}

Cardiac cells were incubated at $37{ }^{\circ} \mathrm{C}$ for different periods of time (24-48 h) with increasing concentrations of the drug and were diluted in DMEM (without phenol red). The mammalian cell morphology and spontaneous contractibility were evaluated by light microscopy, whereas the cellular viability was determined by the colorimetric resazurin assay. After incubation for $24 \mathrm{~h}$, the absorbance was determined at $570 \mathrm{~nm}$ [14]. The obtained data represent the mean of two independent experiments performed in duplicate.

\section{Intracellular amastigotes of $T$. cruzi in peritoneal macrophages}

After the mammalian cytotoxicity studies, the effect of sertraline was investigated against intracellular amastigotes. Peritoneal macrophages $\left(1 \times 10^{5}\right.$ cells/well $)$ were dispensed in 16-well chamber slides (NUNC, Thermo, USA) and maintained for $24 \mathrm{~h}$ in the same medium at $37^{\circ} \mathrm{C}$ in a $5 \%$ $\mathrm{CO}_{2}$ humidified incubator for attachment. Non-adherent cells were removed by two-step washings with medium. After $24 \mathrm{~h}$, these cells were infected with $\left(1 \times 10^{6}\right.$ culture trypomastigote) forms for $4 \mathrm{~h}$ (parasite-to-macrophage ratio 10:1). Subsequently, infected cells were incubated with the drug for $48 \mathrm{~h}$. Finally, the slides were fixed with methanol, stained with Giemsa, and observed via light microscopy. The parasite load was defined by counting 400 macrophages/well by evaluating the number of infected macrophages. Benznidazole was used as the standard drug. The obtained data represent the mean of two independent experiments performed in duplicate.

\section{Intracellular amastigotes of cardiac cell cultures}

For analysis of the effect against intracellular amastigotes from the Y strain, after $24 \mathrm{~h}$ of parasite-host cell interaction, the infected cardiac cell cultures were washed to remove free parasites and were then incubated for another $48 \mathrm{~h}$ with increasing concentrations of the drug.
Cardiac cell cultures were maintained at $37{ }^{\circ} \mathrm{C}$ in an atmosphere of $5 \% \mathrm{CO}_{2}$ and air, and the medium was replaced every $24 \mathrm{~h}$. Then, untreated and treated infected cardiac cell cultures were fixed and stained with Giemsa solution, and the mean number of infected host cells and mean number of parasites per infected cell were scored. Only characteristic T. cruzi nuclei and kinetoplasts were counted as living parasites since irregular structures may indicate parasites undergoing death. The compound activity was estimated by calculating the infection index (II - percentage of infected cells times the average number of intracellular amastigotes per infected host cell) [15]. The obtained data represent the mean of two independent experiments performed in duplicate.

\section{Intracellular amastigotes inside L929 cell lines}

The effect against intracellular forms was also investigated in L929 cell lineages infected with tissue culture-derived trypomastigotes (Tulahuen strain expressing the Escherichia coli $\beta$-galactosidase gene), employing a parasite-to-host-cell ratio of 10:1. After incubation with the drug for $96 \mathrm{~h}$, the viability of parasites was determined colorimetrically as previously reported [14]. The obtained data represent the mean of two independent experiments performed in duplicate.

\section{Mode of action studies of SERT in T. cruzi Spectrofluorimetric detection of the permeability of the cell membrane}

Culture trypomastigotes were washed with PBS (phosphate buffered saline), deposited on a microplate $(2 \times$ $10^{6}$ cells/well) and incubated with SYTOX Green ${ }^{\circ}$ $(1 \mu \mathrm{M})$ for $15 \mathrm{~min}$ at $24{ }^{\circ} \mathrm{C}$ [16]. Sertraline was added at the $\mathrm{IC}_{50}$ value $(2 \mu \mathrm{M})$, and the fluorescence was measured after 20, 40 and $60 \mathrm{~min}$. The maximum permeability was observed with $0.1 \%$ Triton $\mathrm{X}-100$ (positive control). The fluorescence intensity was determined using a plate spectrofluorimeter (FilterMax F5 Multi-Mode MicroplateReader-Molecular Devices) with excitation and emission wavelengths of 485 and $520 \mathrm{~nm}$, respectively. Untreated trypomastigotes and $0.5 \%(\mathrm{v} / \mathrm{v})$ DMSO-treated parasites were used in all assays as negative controls. The obtained data represent the mean of two independent experiments performed in triplicate.

\section{Effect of sertraline on the mitochondrial integrity}

Culture trypomastigotes were washed with PBS, deposited on a microplate $\left(2 \times 10^{6}\right.$ cells/well $)$ and incubated with sertraline at the $\mathrm{IC}_{50}$ value $(2 \mu \mathrm{M})$ for $60 \mathrm{~min}$ at $37{ }^{\circ} \mathrm{C}$. MitoTracker Red CM- $\mathrm{H}_{2}$ XROS (500 nM) was added and the incubation continued for $40 \mathrm{~min}$ in the dark. Parasites were washed twice with HBSS (Hanks' Balanced Salt Solution), and the fluorescence was measured using a plate spectrofluorimeter (FilterMax F5 
Multi-Mode Microplate Reader-Molecular Devices) with excitation and emission wavelengths of 540 and $595 \mathrm{~nm}$, respectively [17]. Carbonyl cyanide 4-(trifluoromethoxy)phenylhydrazone (FCCP; $10 \mu \mathrm{M}$ ) was used as a positive control [18]. The obtained data represent the mean of two independent experiments performed in triplicate.

\section{Analysis of reactive oxygen species (ROS)}

Culture trypomastigotes $\left(2 \times 10^{6}\right.$ cells/well $)$ were washed in HBSS (Hanks' Balanced Salt Solution) and incubated with sertraline at the $\mathrm{IC}_{50}$ value $(2 \mu \mathrm{M})$ for $60 \mathrm{~min}$ at $37^{\circ} \mathrm{C} . \mathrm{H}_{2}$ DCF-DA $(5 \mu \mathrm{M})$ was added, and the cells were incubated for $15 \mathrm{~min}$. The fluorescence intensity was detected using a plate spectrofluorimeter (FilterMax F5 Multi-ModeMicroplate Reader-Molecular Devices) at 485 and $520 \mathrm{~nm}$ for excitation and emission, respectively [19]. The obtained data represent the mean of two independent experiments performed in triplicate.

\section{Measurement of cellular ATP content}

The intracellular adenosine triphosphate (ATP) content was measured by a luciferin-luciferase bioluminescence assay using a specific kit (Life Technologies, USA) according to the manufacturer's instructions. ATP concentrations were calculated using the ATP standard curve kit. Culture trypomastigotes were washed twice with PBS, seeded at $\left(2 \times 10^{6}\right.$ cells/well $)$ and incubated at the $\mathrm{IC}_{50}$ value $(2 \mu \mathrm{M})$ of sertraline in the presence or absence (control) of $0.1 \%$ Triton X-100 for $1 \mathrm{~h}$. The ATP level was rapidly measured by a coupled luciferin-luciferase reaction [20]. The obtained data represent the mean of two independent experiments performed in triplicate.

\section{In silico studies \\ Prediction of sertraline targets using publicly available databases}

We carried out a literature search using PubMed, PubChem Bioassay, ChEMBL and BindingDB in order to identify all possible SERT targets for all organisms. Our definition of a "sertraline target" embraces in vitro assays of SERT against any enzyme, receptor or channel with inhibition $\leq 40 \mu \mathrm{M}$. Then, individual information for each SERT target (primary amino acid sequence in FASTA format, target name and organism) was obtained from the UNIPROT database and was subsequently allocated into a single Excel file (Additional file 2).

\section{Pairwise protein alignment}

Superimposed structures allow a comparison of functionally relevant features, conserved residues necessary for catalysis, and residues critical for ligand binding. Therefore, SERT targets were aligned with all T. cruzi proteins using pairwise BLAST. We considered the T. cruzi target to be druggable if it has $\geq 80 \%$ overlap of the corresponding SERT target and an expected value (E-value) $\leq 10^{-20}$. The E-value represents the number of hits with an alignment score "Z" equal to or greater than the "Z" that would be expected by chance when searching a database, which is the expected number of times a homology will occur at random from a given set of trials.

\section{Comparison of functional regions}

The ConSurf server [21] is a bioinformatics tool for estimating the evolutionary conservation of amino-acid positions in a protein based on the phylogenetic relations between homologous sequences. We used ConSurf for an additional characterization of the functional regions (active site conservation) in T. cruzi targets. Therefore, the degree of conservation of the amino acids from the active site was estimated using 150 homologue proteins with similar sequences retrieved from the UNIPROT database and was identified by the PSI-BLAST method (E-value cutoff $\leq 1^{-10}$ ) [22]. The sequences were clustered, and highly similar (> 95\%) sequences were removed using CD-HIT [23]. A multiple sequence alignment (MSA) of the homologous sequences was constructed using MAFFT-L-INS-I [23]. Subsequently, the MSA was employed to construct a phylogenetic tree using the neighbor-joining algorithm [24]. Position-specific conservation scores were analyzed using the empirical Bayesian method. Subsequently, functional regions were visually compared with corresponding SERT targets and were classified as conserved $(\geq 70 \%)$ or non-conserved $(<70 \%)$. The obtained results are described in Additional files 3, 4, 5 and 6.

\section{Homology modeling and molecular docking}

In the absence of available experimental data, a homology model of $T$. cruzi isocitrate dehydrogenase 2 (TcIDH2) was constructed using homology modeling by comparing the sequence of this target protein with sequences of other proteins (template) for which experimental structures are available. The TcIDH2 sequence was obtained from the TriTrypDB database (Accession: Tc00.1047053506925.319). A BLAST search was carried out with the Protein Data Bank for the identification of a template structure. Three IDH crystal structures were found: human (HsIDH2), Sus scrofa and Mycobacterium tuberculosis (PDB IDs: 4JA8, 1LWD, 4HCX, respectively). Based on the resolution, the crystal structure of $H s$ IDH2 bound to the allosteric inhibitor AGI-6780 (PDB ID 4JA8) [25], was chosen as a template for homology modeling, which presented $66.5 \%$ of sequence identity in relation to TcIDH2. SWISS-MODEL program [26] was used for generation of the homology model of TcIDH2. The quality of the model was assessed using the programs PROCHECK [27], VERIFY 3D [28] and ERRAT. 
Molecular docking studies were performed to investigate the intermolecular interactions between SERT and the amino-acid residues of $T c \mathrm{IDH} 2$ as well as to predict the binding affinity. The generated homology model of TcIDH2 was imported into Maestro v. 10.0 [29] and was prepared using the Protein Preparation Wizard workflow as follows: hydrogen atoms were added according to Epik v. 2.7 ( $\mathrm{pH} 7.4 \pm 1.0)$ and minimized using the OPLS-2005 force field. Next, the structure of SERT was imported from the ChemSpider database and 300 conformations were generated using OMEGA v. 2.5.1.4 [30]. Subsequently, the conformers had their most favorable ionization state calculated at $\mathrm{pH} 7.4$ using the 'fixpka' function, and AM1-BCC charges were added using QUACPAC v.1.6.3.1 [29]. Before the docking studies, two different grids were defined to include the catalytic site and one allosteric site of $T c \mathrm{IDH} 2$. The catalytic site grid was built with the dimensions of $26.3 \AA \times 15.8 \AA \times 27.9 \AA(x, y$ and $z)$ and a volume of $11,645 \AA^{3}$. The allosteric site grid had the dimensions of $16.3 \AA \times 20.3 \AA \times 19.7 \AA$ and a volume of $6531 \AA^{3}$. Finally, molecular docking of SERT with TcIDH2 was investigated using the software FRED, which is available in OEDocking suite v. 3.0.1 [31] using the high-resolution precision and ChemGauss 4 scoring function.

\section{Statistical analysis}

The obtained data represent the mean of three independent experiments performed in duplicate. The $\mathrm{IC}_{50}$ and $\mathrm{CC}_{50}$ values were calculated using sigmoid dose-response curves generated by the software GraphPad Prism version 5.0 (GraphPad Software, San Diego, CA, USA). The ANOVA test was performed to evaluate the significance $(p<0.05)$ of data.

\section{Results}

\section{Antiparasitic activity and mammalian cytotoxicity of} sertraline

The anti-trypanosomal activity of SERT in cell culture-derived trypomastigotes ( $\mathrm{Y}$ strain) was determined colorimetrically by resazurin. After 24 h, $100 \%$ of the parasites were eliminated, resulting in an $\mathrm{IC}_{50}$ value of $1.8 \mu \mathrm{M}( \pm 0.8)$. Using blood-derived trypomastigotes, SERT showed an $\mathrm{IC}_{50}$ value of $14.2 \mu \mathrm{M}( \pm 5.5)$ (Table 1 ).

The drug was also effective against the intracellular amastigotes in peritoneal macrophages, resulting in an $\mathrm{IC}_{50}$ value of $\left.1.4 \quad \pm \quad \pm .6\right) \mu \mathrm{M}$; using intracellular amastigotes in cardiac cell cultures, the $\mathrm{IC}_{50}$ value was $6.6 \mu \mathrm{M}( \pm 1.4)$. The intracellular amastigotes of the / a Tulahuen strain inside fibroblasts (L929 cells) were also susceptible, resulting in an $\mathrm{IC}_{50}$ value of $10.0 \mu \mathrm{M}( \pm 1.7)$ (Table 1). The mammalian cytotoxicity was determined in L929 cells and cardiac cell cultures, and resulted in respective $\mathrm{CC}_{50}$ values of $11.5 \mu \mathrm{M}( \pm 2.48)$ and $25.0 \mu \mathrm{M}$ ( \pm 13.2). Benznidazole was used as the standard and resulted in $\mathrm{IC}_{50}$ values of $17.7 \mu \mathrm{M}( \pm 1.9)$ against trypomastigotes ( $\mathrm{Y}$ strain) and $5 \mu \mathrm{M}( \pm 1.5)$ against intracellular amastigotes (Y strain) (Table 1).

\section{Action mode studies of SERT in $T$. cruzi Permeability of plasma membrane}

To evaluate the possible effect of SERT on the plasma membrane permeability of culture trypomastigotes of $T$. cruzi, SERT was incubated for $60 \mathrm{~min}$ with trypomastigotes at the corresponding $\mathrm{IC}_{50}$ value, after which the permeability of the membrane was evaluated fluorimetrically using the vital dye SYTOX Green ${ }^{\circ}$. The data demonstrated no increase in the fluorescence levels, suggesting that sertraline did not interfere with the plasma membrane permeability of the parasite. Triton $\mathrm{X}-100$ was used as a positive control (data not shown).

\section{Effects on mitochondrial integrity and ATP production}

The mitochondrial integrity of trypomastigotes was investigated in the presence of SERT at the $\mathrm{IC}_{50}$ value using the fluorescent probe Mitotracker Red ${ }^{\circ}$. After $60 \mathrm{~min}$ of incubation, SERT induced an intense and significant depolarization $(p<0.05)$, with the fluorescence intensity decreasing by $100 \%$ when compared to untreated trypomastigotes (control) (Fig. 1a). FCCP was used as a positive control and reduced the fluorescence levels by approximately 54\% compared to untreated parasites. The production of ATP by trypomastigotes was investigated in the presence of SERT using a luminescent assay with luciferase (ATP kit - Thermo). At $60 \mathrm{~min}$ of incubation, an intense decrease in ATP levels of approximately $70 \%$ was observed compared to untreated parasites $(p<0.05)$ (Fig. 1b). Sodium azide was used as a positive control and resulted in a $100 \%$ decrease in the ATP levels of trypomastigotes.

\section{Reactive oxygen species (ROS) production}

Considering the interference of SERT in mitochondrial metabolism, the ROS production by trypomastigotes was

Table 1 Antiparasitic activity and mammalian cytotoxicity of sertraline

\begin{tabular}{|c|c|c|c|c|c|c|c|}
\hline \multicolumn{6}{|c|}{ T. cruzi $\mathrm{IC}_{50} \mu \mathrm{M}( \pm \mathrm{SD})$} & \multicolumn{2}{|c|}{$\mathrm{CC}_{50} \mu \mathrm{M}( \pm \mathrm{SD})$} \\
\hline Drugs & Trypomastigote $(\mathrm{Y})$ & Trypomastigote BT $(\mathrm{Y})$ & Amastigote L929 (Tulahuen) & Amastigote CC $(\mathrm{Y})$ & Amastigote $M \varnothing(\mathrm{Y})$ & L929 & CC \\
\hline SERT & $1.8 \pm 0.8$ & $14.2 \pm 5.5$ & $10.0 \pm 1.7$ & $6.6 \pm 1.4$ & $1.4 \pm 0.6$ & $11.5 \pm 2.4$ & $25.0 \pm 13.2$ \\
\hline$B Z$ & $17.7 \pm 1.9$ & $11.5 \pm 1.0$ & $2.5 \pm 0.9$ & $3.6 \pm 1.0$ & $5.0 \pm 1.5$ & $190.6 \pm 13.4$ & $>200$ \\
\hline
\end{tabular}

$I C_{50} 50 \%$ inhibitory concentration, $C C_{50} 50 \%$ cytotoxic concentration, $C C$ cardiac cells, SERT sertraline, $B Z$ benznidazole, SD standard deviation, $B T$ bloodstream, $M \varnothing$ peritoneal macrophage 


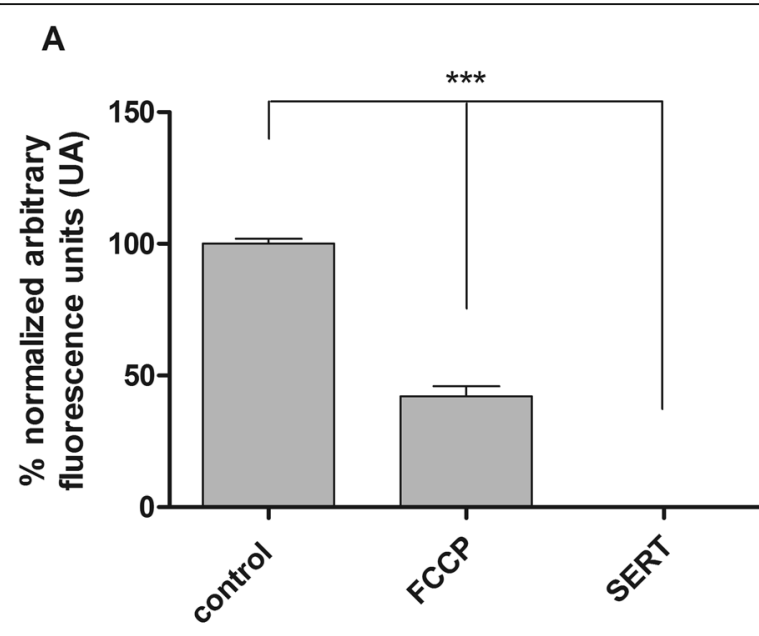

B

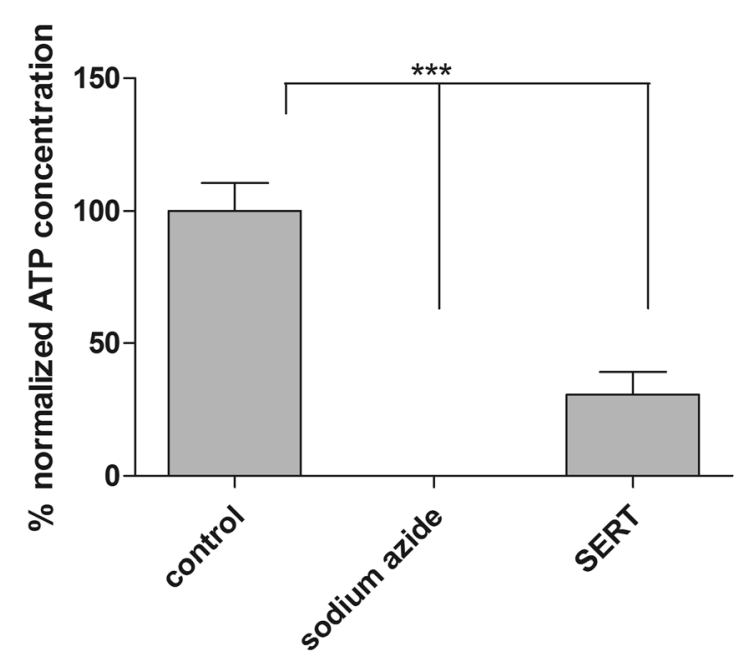

Fig. 1 Evaluation of the bioenergetic parameters of T. cruzi. a Mitochondrial membrane potential of T. cruzi trypomastigotes treated with sertraline for $1 \mathrm{~h}$ at $2 \mu \mathrm{M}$, positive control (FCCP) and negative control (untreated cells). The fluorescence was determined using a fluorimetric microplate reader (FilterMax F5 Multi-Mode Microplate Reader) at 540 and $595 \mathrm{~nm}$ for excitation and emission, respectively. *** $(p<0.05)$; $\mathbf{b}$ Evaluation of the ATP levels of T. cruzi incubated for $1 \mathrm{~h}$ with sertraline at $2 \mu \mathrm{M}$. The levels were measured in a spectroluminometer plate reader (FilterMax F5 Multi-Mode Microplate Reader) using the ATP determination Kit (Life technologies). ${ }^{* *} p<0.05$

evaluated using the fluorescent probe $\mathrm{H}_{2}$ DCf-DA in the presence of the drug. The results indicated no alterations in ROS levels after 60 or 120 min compared to untreated parasites. Oligomycin was used as a positive control, and it upregulated ROS levels in trypomastigotes, as indicated by increased fluorescence intensity (data not shown).

\section{In silico studies}

To identify SERT targets that were experimentally determined in other organisms, we performed a literature search in PubMed, PubChem Bioassay, BindingDB and ChEMBL. Using a chemogenomic target fishing strategy, we identified 15 similar targets in T. cruzi (Additional file 2). Following these conditions, we identified three potential targets of SERT in T. cruzi (Table 2).

\section{Homology modeling and molecular docking}

To build the homology model of TcIDH2, the following criteria were used for template selection: the template should have high coverage, good sequence identity and good X-ray crystallography resolution $(<2.0 \AA)$. Therefore, the selected template, $H s$ IDH2 (PDB ID: 4JA8), presented $66.5 \%$ sequence identity with $T c \mathrm{IDH} 2$, coverage of 0.99 and a resolution of $1.5 \AA$. The stereochemical quality of the TcIDH2 model was evaluated using PROCHECK. This analysis revealed that $91.7 \%$ of residues were within most favored regions, $8.2 \%$ of residues were in additional allowed regions, and only $0.1 \%$ of residues were in disallowed regions of the Ramachandran plot, demonstrating the good quality of the generated model (Fig. 2).

Additionally, the quality of the homology model was evaluated using VERIFY 3D, through which the TcIDH2 model presented a score of $92.6 \%$. An overall quality factor of $86.6 \%$ was obtained from the analysis on ERRAT. The homology model of TcIDH2 had a satisfactory quality and could be used in molecular docking studies. Figure 3a shows the generated 3D structure of the TcIDH2 homology model. Docking studies were performed at two different binding sites of $T c \mathrm{IDH} 2$, the catalytic site, i.e., the binding site of the substrate isocitrate, and in the allosteric site, which is located in the interface between the two chains of the homodimeric protein. The predicted binding affinities of SERT in the catalytic and allosteric sites were $-5.5 \mathrm{kcal} / \mathrm{mol}$ and $-12.2 \mathrm{kcal} / \mathrm{mol}$, respectively. The remarkable difference in binding affinities indicates that SERT may be an allosteric inhibitor of $T c \mathrm{IDH} 2$.

Moreover, docking results showed that the predicted binding mode of SERT at the TcIDH2 allosteric site is in agreement with the experimental $\mathrm{X}$-ray binding mode of the co-crystalized ligand on $H s$ IDH2. In particular, SERT can establish a hydrogen bond between its secondary amine hydrogen and the amino acid residue Gln276A. Additional hydrophobic interactions were observed between the SERT aromatic ring and residues Trp266B, Trp124B and Leu120B (Fig. 3b). The presence of a hydrophobic pocket represented by Leu120A, Leu120B, Trp124B, Ile128B, Ile130B and Trp266B can provide hints for the structural optimization of SERT, thus enabling the design of new inhibitors of TcIDH2.

\section{Discussion}

Drug repurposing is a successful approach with numerous advantages, including reduced time and costs in the drug discovery process. Sertraline, an FDA-approved 
Table $\mathbf{2}$ List of potential sertraline targets in T. cruzi

\begin{tabular}{llllll}
\hline SERT target & T. cruzi target & Biological process & E-value & Overlap & Active Site Conservation (\%) \\
\hline Isocitrate dehydrogenase 1 & Isocitrate dehydrogenase 2 & isocitrate metabolism & 0 & $100 \%$ & High conservation (97\%) \\
Ubiquitin-conjugating enzyme & Ubiquitin-conjugating enzyme & ubiquitin cycle & $1^{-68}$ & $96 \%$ & High conservation (76\%) \\
E2 N & E2, purative & & & High conservation (78\%) \\
Cyclin-dependent kinase 1 & $\begin{array}{l}\text { CDC2-related protein kinase 1, } \\
\text { putative }\end{array}$ & protein phosphorylation & $2^{-113}$ & $95 \%$ & \\
\hline
\end{tabular}

drug, belongs to the class of antidepressant agents known as selective serotonin-reuptake inhibitors (SSRIs). The literature reports several attempts to repurpose the drug SERT towards a range of microorganisms [32, 33].

Considering the broad range of biological activities of SERT, our work investigated the in vitro anti-T. cruzi efficacy against trypomastigotes and intracellular amastigotes. Based on the $50 \%$ Inhibitory Concentration $\left(\mathrm{IC}_{50}\right)$, SERT demonstrated similar in vitro efficacy to the standard drug (BZ) against trypomastigotes and intracellular amastigotes (Y strain). The drug effectively eliminated the intracellular amastigotes of different strains and DTUs of T. crui [34]. It was active against the Y strain (DTU II) in macrophages and cardiac cells at micromolar concentrations. Sertraline was also effective against intracellular amastigotes of the Tulahuen strain (DTU VI), an important feature for a hit compound. Also, it is important to note that the difference between these DTUs may vary in activity and therefore should be taken into account in the testing of new drugs [35]. The Y strain was approximately 7 -fold more susceptible to SERT than the Tulahuen strain. In addition to its intracellular activity against amastigotes, SERT has been valued as a hit candidate for presenting activity against the trypomastigote forms. Katsuno and co-workers [36] stated that drugs that only target the replicating stages of the parasite may leave non-replicating forms, such as trypomastigotes, capable of maintaining infections long after the end of the treatment; i.e., they are a clinical form responsible for relapses.

One of the advantages of repositioning FDA-approved drugs is the existing medical data on the drug, including dosing, side-effects, tolerance profiles and the pharmacokinetic and pharmacodynamics parameters (PKPD). Although sertraline demonstrated in vitro activity and selectivity against $T$. cruzi in our study, one should consider that for in vivo efficacy, adequate tissue distribution and serum levels must be achieved to eliminate the parasite. In the literature, SERT administered in humans resulted in serum levels of approximately $58 \mathrm{nM}$ [37], a considerably smaller value than those needed to eliminate $T$. cruzi intracellular amastigotes (or bloodstream trypomastigotes) in our in vitro assays. Future experimental association studies should be performed to assess

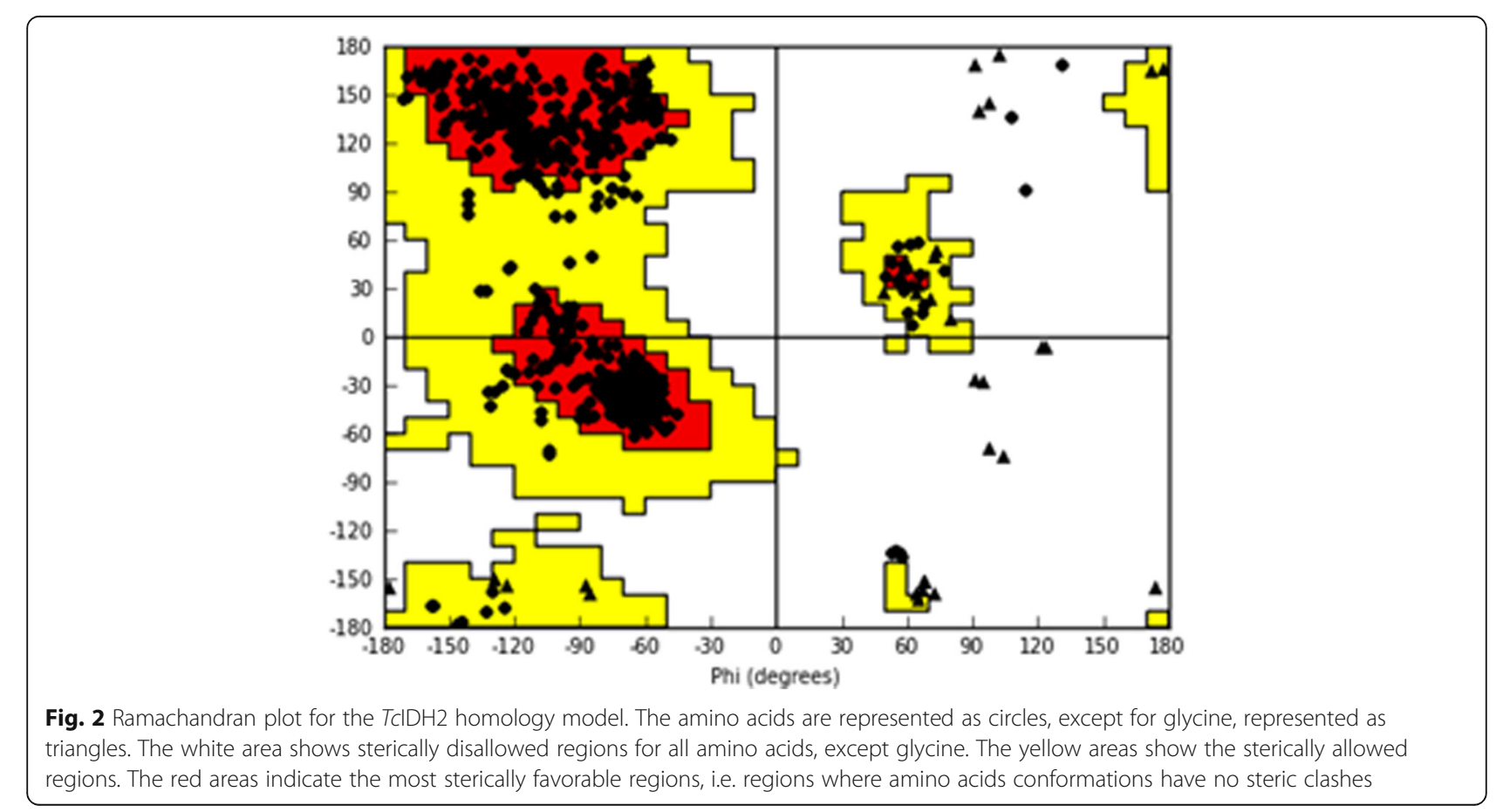




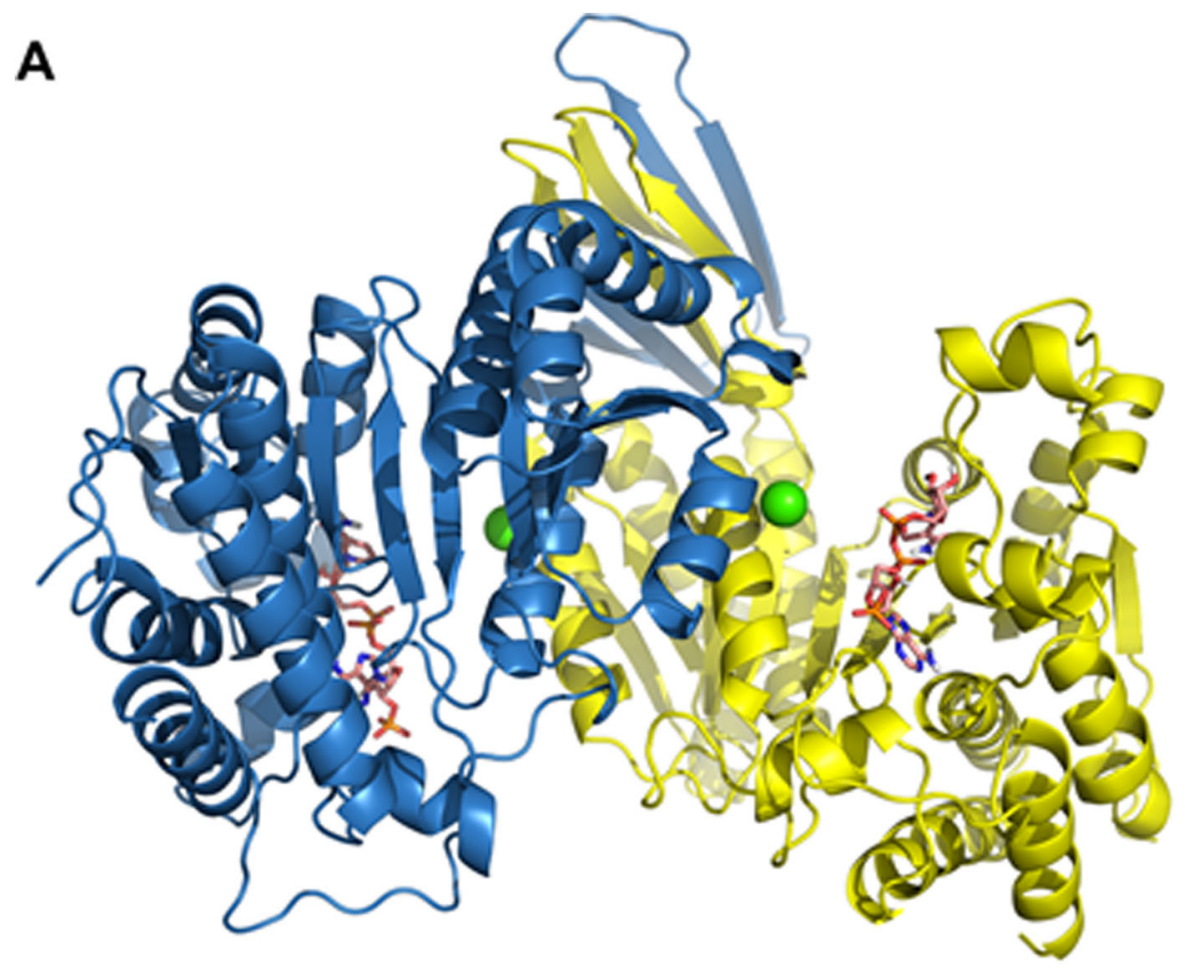

B

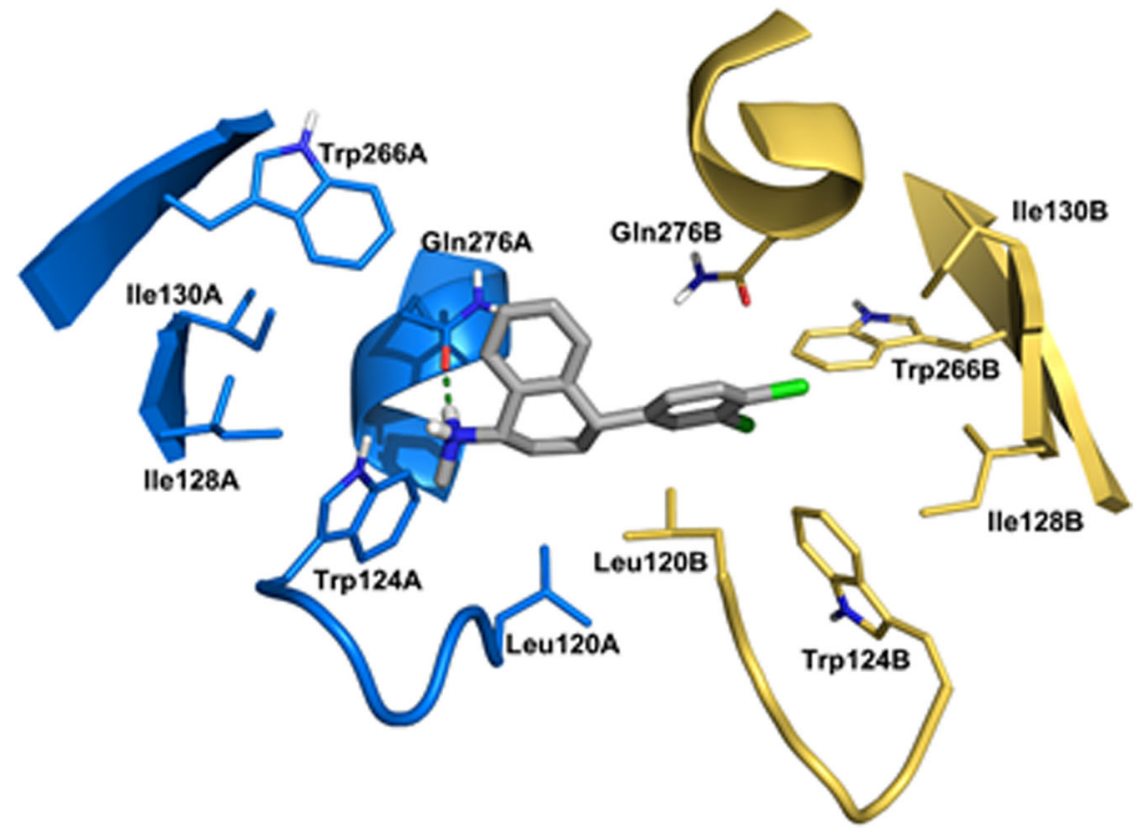

Fig. 3 3D structure of $T \mathrm{CIDH} 2$ homology model. a Regions in blue represent Chain A and regions in yellow represent Chain $\mathrm{B}$. The $\mathrm{Ca}^{2+}$ ions are indicated by green spheres. The two ligands presented in the stick model represent the co-factor NADP. $\mathbf{b}$ Interactions of sertraline with the allosteric binding site of $\mathrm{T} \mathrm{CIDH} 2$ were predicted by docking. The residues of chain A are in blue and chain $\mathrm{B}$ is colored in yellow, indicating the interface that constitutes the allosteric binding site. In the center, sertraline carbon atoms are colored gray, nitrogen in blue, chlorine atoms are green and hydrogens are white. The hydrogen bond between sertraline and Gln276A is indicated by a green dashed line 
sertraline and benznidazole; if synergic combinations are found, reduced doses may be required to treat $T$. cruzi infected animals. Our data also suggest that sertraline may be a useful candidate for both acute and chronic phases of the disease, as the drug is effective against bloodstream trypomastigotes and intracellular amastigotes. However, considering that sertraline is metabolized to desmethylsertraline in the liver, the in vivo efficacy of the drug may be evaluated in the future using both mouse models (acute and chronic).

Considering the promising in vitro efficacy of SERT in T. cruzi parasites, we investigated the possible mode of action using different approaches, including cell biology techniques and in silico approaches. Using short-time incubations to evaluate initial cellular damage in T. cruzi, SERT induced a change in the mitochondrial integrity of trypomastigotes, resulting in a significant decrease in ATP levels within $1 \mathrm{~h}$ without affecting plasma membrane permeability. The depletion of ATP is an early event in mitochondrial dysfunction, which generates irreversible damage and cell death [38].

Trypanosomatids display a single mitochondrion with several peculiar features, such as the presence of different energetic and antioxidant enzymes and a specific arrangement of mitochondrial DNA (kinetoplast DNA) [39]. Due to mitochondrial differences between mammals and trypanosomatids, this organelle is a favorable candidate for drug intervention. Multiple mechanisms and targets are often involved in drug- or xenobiotic-associated mitochondrial impairment [40].

In protozoan parasites such as $L$. donovani, the action mode of sertraline was attributed to the decreased cytoplasmic ATP levels and oxygen consumption rate in promastigotes, suggesting an apoptosis-like death in treated parasites [11]. Previous studies have suggested that the decrease in oxygen consumption and reduction in cytoplasmic ATP levels, resulting from the inhibition of the respiratory chain, are essential events at the beginning of apoptosis in $L$. donovani [41-44]. The effect of sertraline in mitochondria was also demonstrated in hepatocytes. According to Li and co-workers [45], SERT began to decrease ATP levels in as early as $30 \mathrm{~min}$ in rat primary hepatocytes, targeting complexes I and V in the mitochondria. These data also corroborate a previous study of SERT that resulted in concentration-dependent mitochondrial swelling in hepatocytes [45]. In our study, SERT also seems to affect the mitochondria of T. cruzi. Whereas SERT disturbs the bioenergetic system of eukaryotic cells, including Leishmania, the standard drug benznidazole demonstrates a different action mode in T. cruzi. It is important to note that the action mode of BZ is apparently related to the formation of free radicals and electrophilic metabolites that are generated when its nitro-group is reduced to an amino group by the action of nitroreductases [46, 47]. Thus, it is hypothesized that the trypanocidal effect of $\mathrm{BZ}$ is caused by the covalent attachment of its reduced metabolites to macromolecules of the parasite [48].

Currently, several in silico chemogenomic strategies have been applied to drug repositioning against parasitic diseases. The main goal of the chemogenomic approach is to identify new therapeutic targets and drugs. To achieve that goal, various public drug databases that integrate information about gene/protein-drug-disease interactions, such as the Therapeutic Target Database (TTD), DrugBank and STITCH, are valuable resources to develop these strategies. Based on the concept that "similar targets have similar ligands", homology-based inverse virtual screening allowed the prediction of potential targets of SERT in T. cruzi [49].

To identify SERT targets that were experimentally determined in other organisms, we performed a target fishing approach using a literature search in PubMed, PubChem Bioassay, BindingDB and ChEMBL. Using this chemogenomic strategy, we identified 15 similar targets in T. cruzi (Additional file 2). Most target databases are only starting to emerge, and the predicted $T$. cruzi targets are not yet scored for druggability. The druggability concept adds a structural dimension and evaluates the likelihood that small drug-like molecules can bind a given target with sufficient potency to alter its activity [50]. Therefore, the predicted $T$. cruzi targets were considered druggable if they presented an overlap $\geq 80 \%$ of the SERT target, an E-value $\leq 10^{-20}$ and conservation of the functional regions. Thus, overlap sequences and conserved functional regions analysis of positions among SERT targets and T. cruzi targets revealed the importance of each position for protein function and also the possible preservation of affinity for SERT.

Among the predicted targets, isocitrate dehydrogenase 2 (IDH2; Accession: Tc00.1047053506925.319), an enzyme that catalyzes the oxidative decarboxylation of isocitrate to produce 2-oxoglutarate, $\mathrm{CO}_{2}$ and $\mathrm{NADPH}$ [51], was suggested. A search of the T. cruzi genome database (http://www.genedb.org/Homepage) showed the presence of two IDHs, namely IDH1 and IDH2. Both IDHs exhibited remarkable relatedness (>65\% identity) and revealed an equivalent degree of similarity compared to the NADP-linked mammalian counterparts, such as human IDH1. In T. cruzi, IDH1 is restricted to the tricarboxylic acid cycle (Krebs cycle) and provides a portion of the NADH utilized for ATP production by oxidative phosphorylation [52]. In contrast, NADP-linked IDH2 localizes in the peroxisomes, mitochondria and cytosol, where their biological roles are associated with multiple functions, such as intracellular redox homeostasis, $\beta$-oxidation of fatty acids, and lipogenesis. Furthermore, the expression levels of IDH2 are 
significantly increased in amastigotes and trypomastigotes compared to epimastigotes. Conversely, IDH1 appears to be more abundant in the insect stage of T. cruzi [51]. Therefore, our in silico study suggests that SERT is able to inhibit T. cruzi IDH2, a homologue of human IDH1, with higher overlap and conservation of the active site than the other predicted targets.

In addition to the targets highlighted above, 15 other T. cruzi targets were similar to the SERT targets in other organisms. In all cases, we considered the numerical parameters (overlap, conserved functional regions, and E-value) for target homology to be sufficiently significant to infer the predicted target with a high degree of confidence. They were not discussed in detail due to their low druggability profile. For example, T. cruzi mitogen-activated protein kinase (E-value $=5^{-91}$; overlap $=95 \%$ ) has low conservation of functional regions (61\% of predicted residues are conserved), which is insufficient to infer the predicted target with a satisfactory degree of confidence.

\section{Conclusions}

Our studies demonstrated that SERT has a rapid and lethal effect on different forms and strains of T. cruzi, affecting the bioenergetic metabolism of the parasite. The depletion of ATP levels in trypomastigotes and the change in mitochondrial integrity may be initial effects of the drug, contributing to the death of T. cruzi. However, considering the multi-target characteristic of SERT, our in silico studies also suggested that sertraline affects the parasite $T c \mathrm{IDH} 2$, a parasitic enzyme possibly involved in the mechanisms of resistance to oxidative stress. Due to the high homology between the TcIDH1 and TcIDH2, SERT may affect both enzymes. Therefore, further in vitro enzymatic studies involving the predicted enzymes are required to confirm the potential inhibitory effect of SERT. Considering our in vitro experimental studies and the homology-based inverse virtual approach, sertraline should be considered a new hit compound for T. cruzi. These findings provide a starting point for future experimental assays and may contribute to the development of new compounds.

\section{Additional files}

Additional file 1: Flowchart of the global methodology (PNG $147 \mathrm{~kb}$ ) Additional file 2: List of compiled and predicted T. cruzi targets (XLSX $166 \mathrm{~kb}$ )

Additional file 3: Alignment and functional region analysis of the $T$. cruzi IDH2, purative (Sbjct) and human IDH1 (Query) (PNG 312 kb)

Additional file 4: Alignment and functional region analysis of the $T$. cruzi ubiquitin-conjugating enzyme E2, putative (Sbjct) and human ubiquitin-conjugating enzyme E2 N (Query) (PNG 143 kb)
Additional file 5: Alignment and functional region analysis of the $T$. cruzi CRK1 (Sbjct) and human cyclin-dependent kinase 1(Query) (PNG $238 \mathrm{~kb}$ )

Additional file 6: Alignment and functional region analysis of the $T$. cruzi mitogen-activated protein kinase (Sbjct) and human mitogenactivated protein kinase 1 (Query) (PNG 277 kb)

\section{Abbreviations}

ATP: Adenosine triphosphate; BT: Bloodstream trypomastigotes; BZ: Benznidazole; CC: Cardiac cell; CC $_{50}$ : 50\% cytotoxic concentration; DMSO: Dimethyl sulfoxide; DNDi: Drugs for Neglected Diseases Initiative; FBS: Fetal bovine serum; FCCP: Carbonyl cyanide 4-

(trifluoromethoxy)phenylhydrazone; FIOCRUZ: Fundação Oswaldo Cruz; HBSS: Hanks' Balanced Salt Solution; $\mid \mathrm{IC}_{50}$ : $50 \%$ inhibitory concentration; NTDs: Neglected Tropical Diseases; PBS: Phosphate-buffered saline; ROS: Reactive oxygen species; RPMI: Roswell Park Memorial Institute Medium; SERT: Sertraline; TTD: Therapeutic Target Database

\section{Acknowledgements}

We thank Ms. Matilia Ana do Nascimento and Mr. Vicente Duarte for laboratory assistance. We are also grateful to OpenEye Scientific Software, Inc., for providing academic licenses for their software to us.

\section{Funding}

The authors thank São Paulo Research Foundation (FAPESP) for financial support (grant 2015/23403-9 for AGT); Conselho Nacional de Desenvolvimento Científico e Tecnológico (CNPq) for scientific awards to AGT, CHA, MNCS; and Coordenação de Aperfeiçoamento de Pessoal de Nível Superior (CAPES) and Fundação de Amparo à Pesquisa do Estado de Goiás(FAPEG) for financial support and fellowships. The authors are also grateful to FAPERJ and Fiocruz. MNCS is Cientista do Nosso Estado (CNE FAPERJ). This publication was supported by the Coordination for the Improvement of Higher Education Personnel (CAPES) through Programa Editoração CAPES - Edital No. 13/2016, No. do Auxílio 0722/2017, No. do Processo 88881.142062/2017-01 and from the National Council for Scientific and Technological Development (CNPq) Programa Editorial CNPq/CAPES process No. 26/2017, Proc. No. 440954/2017-7.

\section{Availability of data and materials}

Available by request to the corresponding author.

\section{Authors' contributions}

DDF a PhD student, developed the all experimental work; MMR developed part of the in vitro action mechanism studies; JTM developed part of the in vitro action mechanism studies, TADC-S developed part of the in vitro action mechanism studies; DDGJB developed part of the in vitro studies, CFDS; ANSDG developed part of the in vitro studies; BJN developed the in silico studies, CCM-F developed the in silico studies, MDNCS developed and analyzed the in vitro studies, CHA developed and analyzed the in silico studies and AGT is responsible for the design and general ideation of the work and also analyzed the in vitro studies. All authors read and approved the final manuscript.

Ethics approval and consent to participate

Animal procedures were performed with the approval of the Research Ethics Commission, in agreement with the Guidelines for the Care and Use of Laboratory Animals from the National Academy of Sciences. All procedures carried out at Institute Adolfo Lutz were approved by the Committee for Ethics in the Use of Animals (CEUA 04/2016). All procedures performed at Fiocruz were in accordance with the guidelines established by the Fiocruz Committee for Ethics in the Use of Animals (CEUA LW16/14).

Consent for publication

Not applicable.

Competing interests

The authors declare that they have no competing interests. 


\section{Publisher's Note}

Springer Nature remains neutral with regard to jurisdictional claims in published maps and institutional affiliations.

\section{Author details}

'Instituto Adolfo Lutz, Centre for Parasitology and Mycology, Avenida Dr. Arnaldo 351, $8^{\circ}$ andar, sala 9, CEP, São Paulo, SP 01246-000, Brazil. ²Fundação Oswaldo Cruz, Laboratório de Biologia Celular do Instituto Oswaldo Cruz, Av. Brasil, 4365 Manguinhos, CEP, Rio de Janeiro, RJ 21040-360, Brazil. ${ }^{3} F a c u l d a d e$ de Farmácia, Universidade Federal de Goiás, Rua 240 Setor Leste Universitário, Goiânia, GO 74605170, Brazil.

Received: 3 May 2018 Accepted: 5 October 2018 Published online: 30 October 2018

\section{References}

1. Pereira PCM, Navarro EC. Challenges and perspectives of Chagas disease: a review. J Venom Anim Toxins incl Trop Dis. 2013;19(1):34. https://doi.org/10. 1186/1678-9199-19-34.

2. Gascon J, Bern C, Pinazo MJ. Chagas disease in Spain, the United States and other non-endemic countries. Acta Trop. 2010;115(1-2):22-7.

3. Morillo CA, Marin-Neto JA, Avezum A, Sosa-Estani S, Rassi A Jr, Rosas F, et al. Randomized trial of benznidazole for chronic chagas' cardiomyopathy. N Engl J Med. 2015:373(14):1295-306.

4. Pecoul B, Batista C, Stobbaerts E, Ribeiro I, Vilasanjuan R, Gascon J, et al. The BENEFIT trial: where do we go from here? PLoS Negl Trop Dis. 2016;10(2): e0004343.

5. Tempone AG, Sartorelli P, Mady C, Fernandes F. Natural products to antitrypanosomal drugs: an overview of new drug prototypes for American trypanosomiasis. Cardiovasc Hematol Agents Med Chem. 2007;5(3):222-35.

6. Berman J. Miltefosine, an FDA-approved drug for the "orphan disease", leishmaniasis. Expert Opin Orphan Drugs. 2015;3(6):727-35.

7. Duan W, Peng Q, Masuda N, Ford E, Tryggestad E, Ladenheim B, et al. Sertraline slows disease progression and increases neurogenesis in N171-82Q mouse model of Huntington's disease. Neurobiol Dis. 2008:30(3):312-22.

8. Zhai B, Wu C, Wang L, Sachs MS, Lin X. The antidepressant sertraline provides a promising therapeutic option for neurotropic cryptococcal infections. Antimicrob Agents Chemother. 2012;56(7):3758-66.

9. Munoz-Bellido J, Munoz-Criado S, Garcia-Rodrìguez JA. Antimicrobial activity of psychotropic drugs: selective serotonin reuptake inhibitors. Int J Antimicrob Agents. 2000;14(3):177-80.

10. Tuynder M, Fiucci G, Prieur S, Lespagnol A, Géant A, Beaucourt S, et al. Translationally controlled tumor protein is a target of tumor reversion. Proc Natl Acad Sci U S A. 2004:101(43):15364-9.

11. Palit P, Ali N. Oral therapy with sertraline, a selective serotonin reuptake inhibitor, shows activity against Leishmania donovani. J Antimicrob Chemother. 2008;61(5):1120-4.

12. Meirelles MN, de Araujo-Jorge TC, Miranda CF, de Souza W, Barbosa HS. Interaction of Trypanosoma cruzi with heart muscle cells: ultrastructural and cytochemical analysis of endocytic vacuole formation and effect upon myogenesis in vitro. Eur J Cell Biol. 1986;41(2):198-206.

13. Martins LF, Mesquita JT, Pinto EG, Costa-Silva TA, Borborema SE, Galisteo Junior AJ, et al. Analogues of marine guanidine alkaloids are in vitro effective against Trypanosoma cruzi and selectively eliminate Leishmania (L.) infantum intracellular amastigotes. J Nat Prod. 2016;79(9):2202-10.

14. Romanha AJ, Castro SL, Soeiro Mde N, Lannes-Vieira J, Ribeiro I, Talvani A, et al. In vitro and in vivo experimental models for drug screening and development for Chagas disease. Mem Inst Oswaldo Cruz. 2010;105(2):233-8.

15. da Silva CF, Batista Dda G, Oliveira GM, de Souza EM, Hammer ER, da Silva $P B$, et al. In vitro and in vivo investigation of the efficacy of arylimidamide DB1831 and its mesylated salt form--DB1965--against Trypanosoma cruzi infection. PLoS One. 2012;7(1):e30356.

16. Mesquita JT, da Costa-Silva TA, Borborema SET, Tempone AG. Activity of imidazole compounds on Leishmania (L.) infantum chagasi: reactive oxygen species induced by econazole. Mol Cell Biochem. 2014;389(1-2):293-300.

17. Williams RAM, Smith TK, Cull B, Mottram JC, Coombs GH. ATG5 is essential for ATG8-dependent autophagy and mitochondrial homeostasis in Leishmania major. PLoS Pathog. 2012:8(5):e1002695.

18. Chen L, Liu T, Tran A, Lu X, Tomilov AA, Davies V, et al. OPA1 mutation and late-onset cardiomyopathy: mitochondrial dysfunction and mtDNA instability. J Am Heart Assoc. 2012;1(5):e003012.
19. Ribeiro GA, Cunha-Júnior EF, Pinheiro RO, da-Silva SA, Canto-Cavalheiro MM da Silva AJ, et al. LQB-118, an orally active pterocarpanquinone, induces selective oxidative stress and apoptosis in Leishmania amazonensis. J Antimicrob Chemother. 2013;68(4):789-99.

20. Villa-Bellosta R, Wang X, Millán JL, Dubyak GR, O'Neill WC. Extracellular pyrophosphate metabolism and calcification in vascular smooth muscle. Am J Physiol Heart Circ Physiol. 2011;301(1):H61-8.

21. Ashkenazy H, Erez E, Martz E, Pupko T, Ben-Tal N. ConSurf 2010: calculating evolutionary conservation in sequence and structure of proteins and nucleic acids. Nucleic Acids Res. 2010;38:W529-33.

22. Biegert A, Söding J. Sequence context-specific profiles for homology searching. Proc Natl Acad Sci U S A. 2009;106(10):3770-5.

23. Li W, Godzik A. Cd-hit: a fast program for clustering and comparing large sets of protein or nucleotide sequences. Bioinformatics. 2006;22(13):1658-9.

24. Pupko T, Bell RE, Mayrose I, Glaser F, Ben-Tal N. Rate4Site: an algorithmic tool for the identification of functional regions in proteins by surface mapping of evolutionary determinants within their homologues. Bioinformatics. 2002;18(Suppl 1):S71-7.

25. Wang F, Travins J, DeLaBarre B, Penard-Lacronique V, Schalm S, Hansen E, et al. Targeted inhibition of mutant IDH2 in leukemia cells induces cellular differentiation. Science. 2013;340(6132):622-6.

26. Biasini M, Bienert S, Waterhouse A, Arnold K, Studer G, Schmidt T, et al. SWISS-MODEL: modelling protein tertiary and quaternary structure using evolutionary information. Nucleic Acids Res. 2014;42:W252-8.

27. Laskowski RA, MacArthur MW, Moss DS, Thornton JM. PROCHECK: a program to check the stereochemical quality of protein structures. J Appl Crystallogr. 1993;26:283-91.

28. Bowie JU, Lüthy R, Eisenberg D. A method to identify protein sequences that fold into a known three-dimensional stucture. Science. 1991;253(5016): 164-70.

29. Zhu K, Day T, Warshaviak D, Murrett C, Friesner R, Pearlman D. Antibody structure determination using a combination of homology modeling, energy-based refinement, and loop prediction. Proteins. 2014;82(8):1646-55.

30. OMEGA, v2.5.1.4. https://docs.eyesopen.com/omega/citation.html. Accessed 26 July 2017.

31. McGann M. FRED pose prediction and virtual screening accuracy. J Chem Inf Model. 2011;51(3):578-96.

32. Weeks JC, Roberts WM, Leasure C, Suzuki BM, Robinson KJ, Currey H, et al. Sertraline, paroxetine, and chlorpromazine are rapidly acting anthelmintic drugs capable of clinical repurposing. Sci Rep. 2018:8(1):975.

33. Ren J, Zhao Y, Fry EE, Stuart DI. Target identification and mode of action of four chemically divergent drugs against ebolavirus infection. J Med Chem. 2018;61(3):724-33.

34. Zingales B, Andrade SG, Briones MR, Campbell DA, Chiari E, Fernandes O, et al. A new consensus for Trypanosoma cruzi intraspecific nomenclature: second revision meeting recommends Tcl to TcVl. Mem Inst Oswaldo Cruz. 2009:104(7):1051-4.

35. Zingales B, Miles MA, Moraes CB, Luquetti A, Guhl F, Schijman AG, et al. Drug discovery for Chagas disease should consider Trypanosoma cruzi strain diversity. Mem Inst Oswaldo Cruz. 2014;109(6):828-33.

36. Katsuno K, Burrows JN, Duncan K, Hooft van Huijsduijnen R, Kaneko T, Kita $K$, et al. Hit and lead criteria in drug discovery for infectious diseases of the developing world. Nat Rev Drug Discov. 2015;14(11):751-8.

37. Reis M, Aamo T, Ahlner J, Druid H. Reference concentrations of antidepressants. A compilation of postmortem and therapeutic levels. J Anal Toxicol. 2007;31(5):254-64

38. Vercesi AE, Hoffmann ME, Bernardes CF, Docampo R. ATP and Ca2+ homeostasis in Trypanosoma cruzi. Braz J Med Biol Res. 1993;26(4):355-63.

39. Menna-Barreto RFS, de Castro SL. The double-edged sword in pathogenic trypanosomatids: the pivotal role of mitochondria in oxidative stress and bioenergetics. Biomed Res Int. 2014;2014:614014

40. Labbe G, Pessayre D, Fromenty B. Drug-induced liver injury through mitochondrial dysfunction: mechanisms and detection during preclinical safety studies. Fundam Clin Pharmacol. 2008:22(4):335-53.

41. Bermúdez R, Dagger F, D'Aquino JA, Benaim G, Dawidowicz K. Characterization of mitochondrial electron-transfer in Leishmania mexicana. Mol Biochem Parasitol. 1997;90(1):43-54

42. Sen N, Das BB, Ganguly A, Mukherjee T, Tripathi G, Bandyopadhyay S, et al. Camptothecin induced mitochondrial dysfunction leading to programmed cell death in unicellular hemoflagellate Leishmania donovani. Cell Death Differ. 2004;11(8):924-36. 
43. Mehata A, Shaha C. Apoptotic death in Leishmania donovani promastigotes in response to respiratory chain inhibition: complex II inhibition results in increased pentamidine cytotoxicity. J Biol Chem. 2004;279(12):11798-813.

44. Sen N, Das BB, Ganguly A, Mukherjee T, Bandyopadhyay S, Majumder HK. Camptothecin-induced imbalance in intracellular cation homeostasis regulates programmed cell death in unicellular hemoflagellate Leishmania donovani. J Biol Chem. 2004;279(50):52366-75.

45. Li Y, Couch L, Higuchi M, Fang JL, Guo L. Mitochondrial dysfunction induced by sertraline, an antidepressant agent. Toxicol Sci. 2012;127(2):582-91.

46. Maya JD, Cassels BK, Iturriaga-Vásquez P, Ferreira J, Faúndez M, Galanti N, et al. Mode of action of natural and synthetic drugs against Trypanosoma cruzi and their interaction with the mammalian host. Comp Biochem Physiol A Mol Integr Physiol. 2007;146(4):601-20.

47. Wilkinson SR, Taylor MC, Horn D, Kelly JM, Cheeseman I. A mechanism for cross-resistance to nifurtimox and benznidazole in trypanosomes. Proc Natl Acad Sci U S A. 2008;105(13):5022-7.

48. Maya JD, Rodríquez A, Pino L, Pabón A, Ferreira J, Pavani M, et al. Effects of buthionine sulfoximine nifurtimox and benznidazole upon trypanothione and metallothionein proteins in Trypanosoma cruzi. Biol Res. 2004;37(1):61-9.

49. Rognan D. Chemogenomic approaches to rational drug design. Br J Pharmacol. 2007;152(1):38-52.

50. Neves BJ, Braga RC, Bezerra JCB, Cravo PVL, Andrade CH. In silico repositioning-chemogenomics strategy identifies new drugs with potential activity against multiple life stages of Schistosoma mansoni. PLoS Negl Trop Dis. 2015;9(1):e3435.

51. Leroux AE, Maugeri DA, Cazzulo JJ, Nowicki C. Functional characterization of NADP-dependent isocitrate dehydrogenase isozymes from Trypanosoma cruzi. Mol Biochem Parasitol. 2011;177(1):61-4.

52. Adroher FJ, Osuna A, Lupiañez JA. Differential energetic metabolism during Trypanosoma cruzi differentiation. I. Citrate synthase, NADP-isocitrate dehydrogenase, and succinate dehydrogenase. Arch Biochem Biophys. 1988;267(1):252-61.

Ready to submit your research? Choose BMC and benefit from:

- fast, convenient online submission

- thorough peer review by experienced researchers in your field

- rapid publication on acceptance

- support for research data, including large and complex data types

- gold Open Access which fosters wider collaboration and increased citations

- maximum visibility for your research: over $100 \mathrm{M}$ website views per year

At $\mathrm{BMC}$, research is always in progress.

Learn more biomedcentral.com/submissions 